\title{
Kalzifizierende venös-aneurysmatische Malformation der Thoraxwand als seltene Ursache von Lungenembolien - eine Kasuistik
}

\author{
Venous Aneurysmatic Malformation of the Chest Wall as a Rare Cause \\ of Pulmonary Embolism - A Case Report
}

Autoren

Institute
R. Luther ${ }^{1}$, F. Meyer ${ }^{2}$, J. Ricke ${ }^{3}$, H. Presser ${ }^{4}$, H. Nowak ${ }^{5}$, R. Aisch ${ }^{6}$, H. Lindekamm 6 , Z. Halloul ${ }^{2}$

Die Institutsangaben sind am Ende des Beitrags gelistet. eingereicht 12.3 .2008 akzeptiert nach Revision 21.7.2008

Bibliografie

Dol $10.1055 / \mathrm{s}-2008-1038255$ Online-Publikation: 5. 9. 2008 Pneumologie 2009; 63: 10-13 (c) Georg Thieme Verlag KC Stuttgart · New York ISSN 0934-8387

\section{Korrespondenzadresse} Dr. med. Ronald Luther Lemsdorfer Weg 7 39112 Magdeburg Ronald.Luther@gmx.de

\section{Zusammenfassung \\ $\nabla$}

Es wird über einen 66-jährigen Mann berichtet, bei dem rezidivierende Lungenembolien auftraten. Phlebografisch wurde ein monströses venöses Aneurysma der rechten Thoraxwand in Kombination mit multiplen Venendilatationen und Kalzifizierungen als Emboliequelle verifiziert. Nach interventionell-radiologischer Embolisation und chirurgischer Ligatur des Aneurysmenhalses traten binnen 23 Monaten Nachbeobachtungszeit unter Antikoagulation weitere Embolien nicht mehr auf.

\section{Fallbericht}

Bei einem 66jährigen Betriebshandwerker traten im August 2004 Episoden akuter Dyspnoe mit spontaner Normalisierung auf. Es bestand eine hypotone, jedoch normofrequente Kreislaufregulation (RR 100/60 mm Hg).

Wegen persistierender, akuter Luftnot erfolgte die stationäre Aufnahme in das regionale Kreiskrankenhaus.

Klinisch dominierte zum Zeitpunkt der stationären Aufnahme eine Ruhedyspnoe. Thoraxschmerzen und Auswurf lagen nicht vor.

Laborchemisch bestand eine Leukozytose (13,7 Gpt/l), das CRP war mit $6 \mathrm{mg} / \mathrm{dl}$, die CK mit $3,41 \mu \mathrm{mol} /$ lxs jeweils leicht erhöht. D-Dimer 2,9 $\mathrm{mg} / \mathrm{l}$, Myoglobin 178,8 $\mu \mathrm{g} / \mathrm{l}$; Troponin 0,122 ng/ml. Röntgenologisch zeigte die Thoraxaufnahme keinen pathologischen pulmonalen Befund.

Echokardiografisch normokinetischer, normal großer Ventrikel mit Wandhypertrophie. Rechter Ventrikel vergrößert. Über der Trikuspidalklappe Insuffizienzjet; errechneter systolischer Pulmonalarteriendruck von $65 \mathrm{~mm} \mathrm{Hg}$.

Klinisch und laborchemisch wurde eine akute Lungenembolie diagnostiziert. Nach FibrinolyseTherapie (Rapilysin ${ }^{\circledR}$, Fa. Roche, Grenzach-

\section{Abstract \\ $\nabla$}

This is a case report about a 66-year old man with recurrent pulmonary embolisms. Phlebography revealed a monstrous venous aneurysm of the right chest wall with several venous dilatations and calcification as the source of the embolism. Radiological embolisation and surgical ligation of the large aneurysmatic neck were performed. No further embolism episodes were registered under anticoagulation during the 23 months of the postoperative follow-up investigation.
Wyhlen, BRD) und nachfolgender i.v.-HeparinApplikation wurde eine rasche Stabilisierung der respiratorischen Situation erreicht. Im Weiteren Umstellung auf orale Antikoagulation (Falithrom $^{\circledR}$, Fa. Hexal, Holzkirchen, BRD).

Nebenbefundlich wurden zum Ausschluss eines paraneoplastischen Geschehens weitere Untersuchungen durchgeführt. Die Gastroskopie zeigte eine schwere Antrumgastritis mit mehreren Ulcerationen. Koloskopisch wurden 5 Polypen nachgewiesen und abgetragen (histologisch: tubulovillöse Adenome mit geringgradiger Dysplasie).

Ein sonografisch bestehender Verdacht auf eine Prostata-Neoplasie (bei erhöhtem PSA-Wert) wurde bioptisch nicht bestätigt.

Unter laufender Antikoagulation blieb der Patient nach Entlassung zunächst beschwerdefrei. Wegen peripherer arterieller Verschlusskrankheit (Stadium IV nach Fontaine) mit Gangrän der 3. Zehe rechts wurde im Dezember 2005 die gefäßchirurgische Desobliteration der rechten Beckenachse und des rechten Oberschenkels durchgeführt.

Bei persistierender Belastungsdyspnoe erfolgte eine pneumologische Diagnostik im Mai 2006.

Röntgenologisch waren die Lungenfelder beidseits weitgehend unauffällig. 


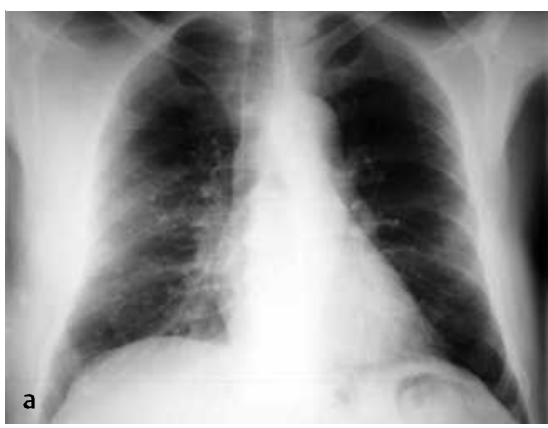

Abb. 1 Röntgen-

Thorax:

a) Verkalkungsstrukturen an lateraler Thoraxwand rechts. b) Frontal: Multiple Rundschatten, vorrangig ventral.

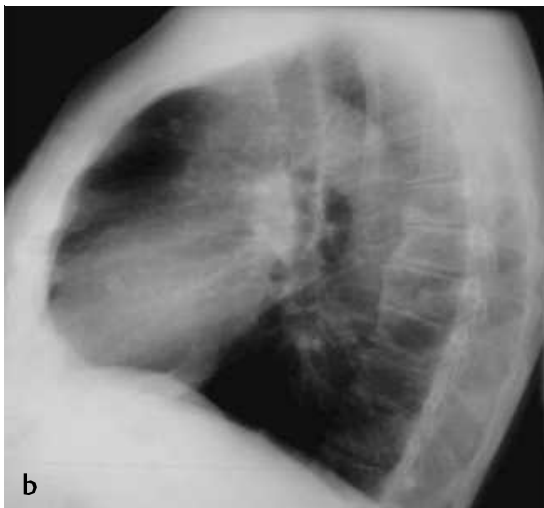

Extrapulmonal fanden sich im Bereich der lateralen Thoraxwand rechts multiple rundliche Schatten mit glatter Begrenzung und partiellen Kalzifizierungen $(\bullet$ Abb. 1 a, b).

Die bodyplethysmografisch ermittelten Funktionsparameter zeigten Normalwerte $\left(\mathrm{FEV}_{1} 3,64 \mathrm{l}=118 \% \mathrm{SW} ; \mathrm{VC}_{\mathrm{IN}} 4,12 \mathrm{l}=100 \%\right.$ SW). Die CO-Diffusionskapazität lag im Normbereich.

Der Methacholin-Test blieb ohne Nachweis einer bronchialen Hyperreaktivität. Im EKG bestanden keine Hinweise auf eine Rechtsherzhypertrophie.

Eine Ergooxytensiometrie wurde nach geringer Belastung $(30 \mathrm{~W}$, 5. Minute) wegen Kräftemangels und Hypertonie abgebrochen (bei maximalem RR von 210/120 mm Hg). Der Ruhe- $\mathrm{pO}_{2}$ wurde mit $88 \mathrm{~mm} \mathrm{Hg}$ bestimmt, bei 30 Watt wurden $80 \mathrm{~mm} \mathrm{Hg}$ registriert.

Eine echokardiografische Kontrolle ergab 07/2006 weitgehend normalisierte Befunde (geringe Trikuspidalklappeninsuffizienz I. Grades); keine pathologisch erhöht geschätzten Druckwerte in der A. pulmonalis.

Wegen der Thoraxwandveränderungen erfolgte eine Spiralcomputertomografie des Thorax. Im CT zeigten sich im Bereich der rechten Thoraxwand - beginnend in der Axilla - multiple, rundliche Verkalkungsfiguren, zum Teil angrenzend an abnorme, varikös erweiterte Gefäßstrukturen sowie eine kolbig-langstreckige Auftreibung eines Gefäßes rechtsaxillär, welches bis weit nach kaudal hin nachweisbar war ( $\bullet$ Abb.2). Die genann- ten Veränderungen wurden als suspekte Emboliequelle eingestuft.

Als Kofaktor für das embolische Geschehen wurde ein thrombophil wirksamer Gerinnungsdefekt (Lupus-Hemmkörper) nachgewiesen.

Die Komplettierung der Diagnostik wurde durch ein erneutes embolisches Ereignis trotz laufender (effektiver) Antikoagulation unterbrochen, das bei therapeutischer Heparinisierung unter stationären Bedingungen zügig zu beherrschen war.

Bei Phlebografie des rechten Armes (Optiray ${ }^{\circledR}$ 300, Fa. Tyco Healthcare, Neustadt/Donau, BRD) über eine Kubitalvene zeigte die V. cephalica keinen Kontrastmittelabfluss in die V. subclavia. Retrograd erfolgte die Auffüllung einer ektatischen V. axillaris sowie der Vv. brachiales et profundae brachii. Zeitgleich stellte sich eine monströs aneurysmatisch erweiterte venöse Struktur der dorsolateralen rechten Thoraxwand unterhalb der Axillarregion - zunächst ohne Abfluss nach zentral - dar ( $\bullet$ Abb. 3 a u. b). Nach kaudal bestanden weitere varikös erweiterte Venen. Erst nach Lageänderung sowie durch Bewegung des Schultergürtels kam es zum regulären Abstrom des Kontrastmittels von der V. axillaris in die V. subclavia. Gleichzeitig entleerte sich der große Aneurysmasack nahezu vollständig in die V. subclavia (๑ Abb.3c u.d).

Das klinisch-pathologische und angiologisch-topografische Bild mit schwerst aneurysmatisch veränderten Venenstrukturen und Verkalkungen wurde als - wahrscheinlich konnatale - Veränderung interpretiert, wobei der große Aneurysmasack mit erheblicher Entleerungsverzögerung als kausalpathogenetischer Faktor für die rezidivierenden Lungenembolien bei zusätzlich vorliegender Thrombophilie angesehen wurde.

07/2006 erfolgte die definitive Therapie der Malformation im Hybridverfahren mittels:

- interventionell-radiologischer Embolisation des venösen Aneurysmas

- Instillation von 40,0 ml Äthanol 95\% (via 5F-Headhunter-Katheter mit selektiver Katheterisierung des Aneurysmasackes, eingeführt über rechtsinguinal in die V. femoralis communis platzierte 5F-Schleuse)

- simultane Blockung mittels Occlusionsballon am Aneurysmahals zur Verhinderung der Abschwemmung von Embolisationsmaterial bzw. zur Thrombembolieprophylaxe, eingeführt über eine 14F-Schleuse, die linksinguinal in der $\mathrm{V}$. femoralis platziert war.

- Nachfolgende Dokumentation des partiellen Thrombosierungserfolges mittels Durchleuchtungskontrolle (ausgedehnter Thrombus von ca. $4 \times 2 \mathrm{~cm}$ Größe)

- offen-gefäßchirurgische Ligatur des Aneurysmahalses unter Schutz des platzierten Occlusionsballons mit nachfolgender schrittweiser Entblockung sowie Entfernung des Ballonkatheters.

Der Eingriff wurde vom Patienten gut toleriert.

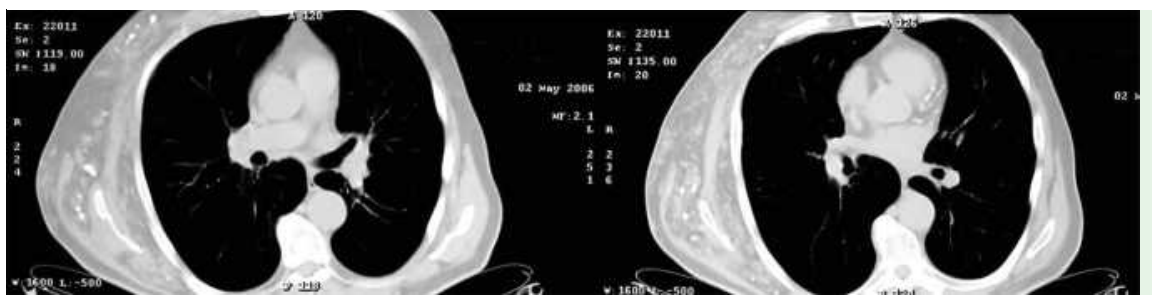

Abb.2 CT des Thorax (Ausschnitt): Verdickung der rechten Thoraxwand mit Nachweis von multiplen Rundschatten mit Kalzifizierungen. 


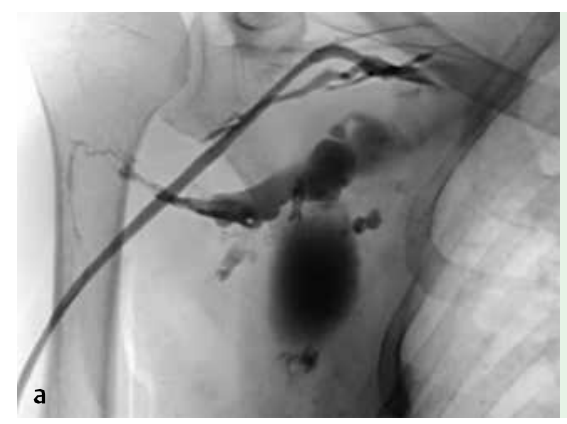

Abb.3a Phlebografie des rechten Armes (unmittelbar nach Kontrastmittel-Injektion): Darstellung der V. brachialis, eines monströsen venösen Aneurysmas der re. Thoraxwand sowie zusätzliche Rundschatten mit Kontrastmittelfüllung.

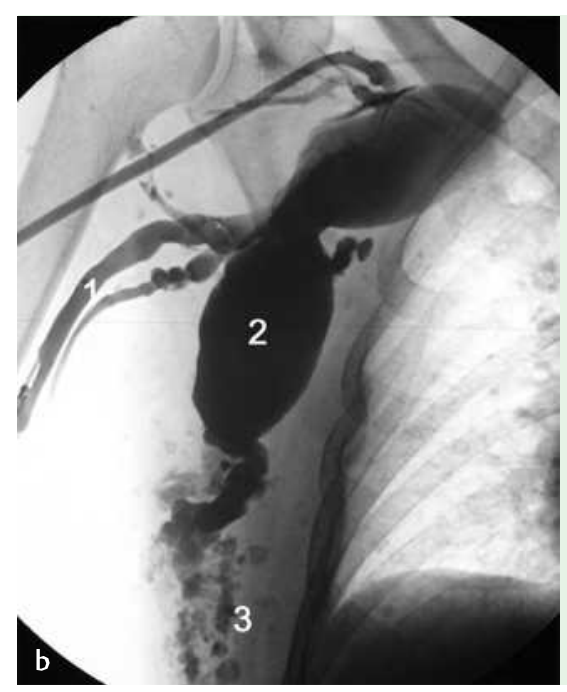
nach KM-Injektion: Großes Aneurysma der Thoraxwand (2), gefüllte, ektatische Venen der Thoraxwand $(1,3)$.
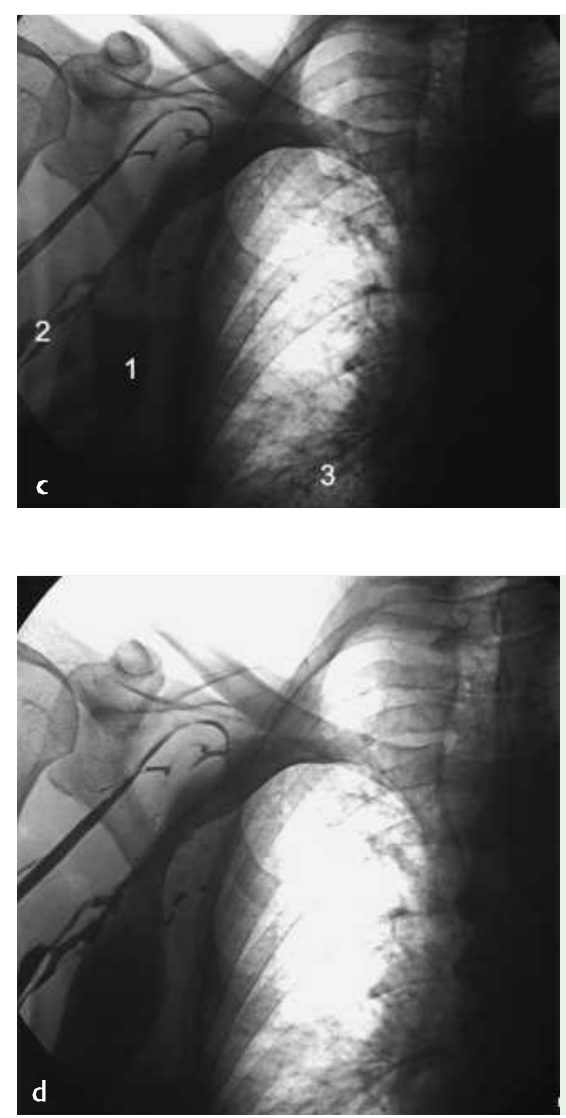

Abb.3c Plötzlicher Kontrastmittelabfluss aus dem Thoraxwandaneurysma (1) und Kollateralen (2) nach Lageänderung; KM-Nachweis in Pulmonalarterien (3).

Abb.3d Spätphase (5. Minute nach KM-Injektion): Allmähliche Entleerung des Aneurysmas.
Abb.3b 2. Minute

Unter Antikoagulantien-Medikation (Falithrom ${ }^{\circledR}$ ) waren im bisherigen Follow-up-Zeitraum von 23 Monaten keine weiteren embolischen Ereignisse zu verzeichnen.

\section{Diskussion \\ $\nabla$}

Aneurysmata des venösen Systems sind seltene vaskuläre Malformationen und treten ohne Geschlechtsdominanz auf. Die Genese ist im Allgemeinen kongenital-idiopathisch.

Darüber hinaus wurden aneurysmatische Deformationen auch posttraumatisch nach stumpfem Thoraxtrauma beschrieben [1]. Als Prädilektionsstellen werden vorrangig untere und obere Extremitäten sowie weitere größere Venen (wie Vv. jugularis, subclavia, brachiocephalica, basilaris, azygos, cava superior et inferior, mesenterica, splenica, iliaca u. a.) beschrieben.

Beim Vorliegen venöser Aneurysmata finden sich häufig bereits in der Adoleszenz oder in frühem Erwachsenenalter thromboembolische Komplikationen, auch Rupturen wurden mitgeteilt [2-17].

Bei intrathorakaler Lage führen Venenaneurysmata vielfach zu Mediastinalveränderungen, die konventionell-radiologisch oder mittels CT und MRT relativ einfach diagnostizierbar sind [18-20].

Gelegentlich wurde über ein gemeinsames Auftreten venöser Aneurysmata mit anderen Malformationen (z. B. V. cava superior sinistra persistens [14] und Erkrankungen wie zystischen Hygromen [21] und Hämangiomen [22]) berichtet.

Zum überwiegenden Teil liegen zu diesen Erkrankungen in der Literatur Einzelfallberichte vor (Chou u. Mitarb. [4]; Gozdziuk u. Mitarb. [20]; Haniuda u. Mitarb. [8]; Picou u. Mitarb. [10]; Regueiro u. Mitarb. [12]; Tsuji u. Mitarb. [15]; Watanabe u. Mitarb. [17]).
In einer Sammelstatistik berichteten Sullivan u. Mitarb. 2002 über 18 Fälle von venösen Aneurysmata in unterschiedlichen Körperregionen [13].

Während arteriovenöse Aneurysmata der Thoraxwand gelegentlich beschrieben wurden $[23,24]$, scheinen rein venöse Malformationen der Thoraxwand noch seltener zu sein.

Therapeutisch wird in der Literatur regelhaft eine prophylaktische Resektion empfohlen, sofern eine Lokalisation abdominal oder im Bereich der unteren Extremitäten vorliegt. Bei Lokalisation in anderen Regionen wird eine Exzision nur angestrebt, wenn die Veränderungen zu Symptomen führen, eine Größenzunahme zeigen oder Entstellungen verursachen [25].

Im präsentierten Fall führte eine primäre Lungenembolie mit postembolisch persistierender Dyspnoe zu weiterführenden Untersuchungen, die den Befund einer monströsen, venös-aneurysmatischen Malformation der Thoraxwand rechts ergaben, welche als Quelle für die Lungenembolien anzusehen war.

Eine traumatisch schonende Kombination aus interventionellradiologischer Embolisation von Aneurysma-Anteilen und chirurgischer Behandlung mittels Ligatur des Aneurysmahalses sicherte im mittelfristigen Follow-up-Intervall den Therapieerfolg im vorgestellten Fall, so dass Embolie-Rezidive unter fortlaufender oraler Antikoagulation vermieden werden konnten.

Im Falle von anspruchsvollen und seltenen gefäßmedizinischen Fallkonstellationen - wie beim vorliegenden, extraordinären Patienten - sind konsequente, interdisziplinäre Diagnosesicherungsstrategien erforderlich. Im Rahmen der Behandlung wird eine interdisziplinäre Therapieplanung in einem Gefäßzentrum 
favorisiert, die eine flexible Methodenvariabilität bei akzeptablem Risiko-Nutzen-Verhältnis ermöglicht.

Die sinnvolle und abgestimmte Abfolge von interventionell-radiologischem und gefäßchirurgischem Procedere ermöglichte in unserem Fall eine patientenschonende Sanierung der Emboliequelle unter Gewährleistung maximaler Sicherheit der Prävention erneuter - möglicherweise vital bedrohlicher - Lungenembolieepisoden bei Vermeidung eines ausgedehnten chirurgischen Traumas infolge Ausschöpfung minimal-invasiver Behandlungsoptionen in einem interdisziplinären Therapiekonzept.

Institute

${ }^{1}$ Pneumologische Praxis, Magdeburg

${ }^{2}$ Klinik für Chirurgie, Otto-von-Guericke-Universität, Magdeburg

(Direktor: Prof. Dr. med. W. Lippert)

${ }^{3}$ Institut für Radiologie, Otto-von-Guericke-Unversität, Magdeburg

(Direktor: Prof. Dr. med. J. Ricke)

${ }^{4}$ Sana Ohre-Klinikum Haldensleben, Innere Klinik

(Chefarzt: Dr. med. H. Presser)

${ }^{5}$ Ohrekreisklinikum, Standort Wolmirstedt, Röntgeninstitut

(amt. Chefarzt: Dipl. med. H. Nowak)

${ }^{6}$ Radiologische Gemeinschaftspraxis, Haldensleben

\section{Literatur}

1 Rappaport DC Jr, Ros PR, Moser RP. Idiopathic dilatation of the thoracic venous system. Can Assoc Radiol J 1992; 43: 385- 387

2 Burkill GJC, Burn PR, Padley SPG. Aneurysm of the left brachiocephalic vein: An unusual cause of mediastinal widening. Brit J Radiol 1997; 70: $837-839$

3 Busch T, Aleksic I, Schulze F et al. Retrocardiac arteriovenous malformation causing recurrent cerebral ischemia. Ann Thorac Surg 2000; 70: $663-665$

4 Chou YP, Hsu HK, Goan YG et al. Aneurysm of superior vena cava: A case report. Formosan J Surg 2000; 33: 304-308

5 Ekim H, Kutay V, Tuncer $M$ et al. Management of primary venous aneurysms. Saudi Med J 2004; 25 (3): $303-307$

6 Galeggo M, Mirapeix RM, Castaner $E$ et al. Idiopathic azygos vein aneurysm: A rare cause of mediastinal mass. Thorax 1999; 54: 653-655

7 Gillespie DL, Villavicencio JL, Gallagher C et al. Presentation and management of venous aneurysms. J Vasc Surg 1997; 26: 845-852
8 Haniuda M, Numanami H, Makiuchi A et al. Solitary aneurysm of the innominate vein. J Thoracic Imaging 2000; 15: 205-207

9 Perrin M. Venous aneurysms. Phlebolymphology 2006; 13: 172-176

10 Picou MA, Antonovic R, Holden WE. Position-dependent mediastinal mass: Aneurysm of the superior vena cava. Am J Roentgenol 1993; 161: $1110-1111$

11 Regina G, Cardia G, Squeo MA et al. Aneurysm of the internal jugular vein. A case report. Vascular Surg 1988; 22: 169-171

12 Regueiro Mira F, Galbis Caravajal JM et al. Thoracic venous aneurysms. Clinical Observation. J Cardiovasc Surg 2002; 43: 527-529

13 Sullivan VV, Voris TK, Borlazza GS et al. Incidental discovery of an inferior vena cava aneurysm. Ann Vasc Surg 2002; 16: 513-515

14 Taira A, Akita $\mathrm{H}$. Ruptured venous aneurysm of the persistent left superior vena cava. Angiology 1981; 32: 656 - 659

15 Tsuji A, Katada Y, Tanimoto $M$ et al. Congenital giant aneurysm of the left innominate vein: is surgical treatment required? Pediatr Cardiol 2004; 4: 421 - 423

16 Uematsu M, Okada $M$. Primary venous aneurysms - case reports. Angiology 1999; 50: 239-324

17 Watanabe A, Kusajima K, Aisaka N et al. Idiopathic saccular azygos vein aneurysm. Ann Thoracic Surg 1998; 65: 1459- 1461

18 Abad Santamaria N, Garcia Diez JM, Pavon Fernandez MJ et al. Azygos vein aneurysm forming a mediastinal mass. Arch Broncopneumol 2006; 42: 410-412

19 Gomez MA, Delhommais A, Presicci PF et al. Partial thrombosis of an idiopathic azygos vein aneurysm. Brit J Radiol 2004; 77: 342 - 343

20 Gozdziuk K, Czekajska-Chehab E, Wrona A et al. Saccular aneurysm of the superior vena cava detected by computed tomography and successfully treated with surgery. Ann Thoracic Surg 2004; 78: e94-e95

21 Joseph AE, Donaldson JS, Reynolds M. Neck and thorax venous aneurysm: Association with cystic hygroma. Radiology 1989; 170: 109112

22 Hammerer I, Stampfel G. Ausgedehntes Haemagioma racemosum des Mediastinums mit Zwerchfellparese. Ein Fallbericht und Beitrag zur Differenzialdiagnose des arteriovenösen Aneurysmas im Thoraxbereich. Paediatr Padol 177; 12: 63 - 72

23 Eloit J, Henry M, Hoeffel JC et al. Aneurysme arterio-veineux congenital de la paroi thoracique. A propos dun cas. J Radiol Electrol Med Nucl 1974; 55: 59-61

24 Heidrich H, Paeprer H, Nasseri $M$ et al. Arteriovenöses Aneurysma zwischen Interkostalarterie und Vena subclavia. Fortschr Röntgenstr Nuklearmed 1975; 123: $45-48$

25 Calligaro KD, Ahmad S, Dandora $R$ et al. Venous aneurysms: surgical indications and review of the literature. Surgery 1995; 117: $1-6$ 